\title{
EDUCAÇÃO, FORMAÇÃO DOCENTE, TDIC E SAÚDE EM TEMPOS DE PANDEMIA PELA COVID-19: UMA REVISÃO DE LITERATURA
}

\author{
EDUCACIÓN, FORMACIÓN DOCENTE, TDIC Y SALUD EM TIEMPOS DE \\ PANDEMIA POR COVID-19: UNA REVISIÓN DE LA LITERATURA
}

\author{
EDUCATION, TEACHER FORMATION, DICT, AND HEALTH IN TIMES OF COVID- \\ 19 PANDEMIC: A LITERATURE REVIEW
}

\author{
Ronaldo Julio BAGANHA ${ }^{1}$ \\ Ana Carolina Brasil e BERNARDES ${ }^{2}$ \\ Lucas Gambogi ANTUNES ${ }^{3}$
}

RESUMO: Este artigo teve como objetivo revisar por meio da literatura os desafios e as transformações impostas pela pandemia da COVID-19 à educação, as principais contribuições da formação docente para inovação nos processos de ensino e de aprendizagem com a utilização das TDIC e as possíveis consequências à saúde do aumento do tempo de tela e elevado comportamento sedentário. Os resultados mostram que diante dos desafios da educação em virtude da pandemia, o domínio e o entendimento dos conhecimentos sobre as TDIC utilizadas no ambiente escolar, são essenciais para os docentes, o que implica na necessidade de cursos de formação de professores que subsidiem a integração dessas tecnologias nos currículos e práticas pedagógicas. Destaca-se ainda que o elevado tempo de exposição à "tela" pode prejudicar a saúde, e assim, faz-se necessária uma orientação aos professores e alunos, no sentido preventivo de danos à saúde mental.

PALAVRAS-CHAVE: Educação. Pandemia. TDIC. Tempo de tela.

RESUMEN: Este artículo tiene como objetivo revisar, através de la literatura, los desafios y transformaciones que la pandemia del COVID-19 impone a la educación, los principales aportes de la formación docente a la innovación en los procesos de enseñanza y aprendizaje con el uso de TDIC y las posibles consecuencias para la salud del aumento. en tiempo de pantalla y alto comportamiento sedentario. Los resultados muestran que, dados los desafios de la educación por la pandemia, el dominio y comprensión del conocimiento sobre el TDIC utilizado en el ámbito escolar son fundamentales para los docentes, lo que implica la necesidad de cursos de formación de docentes que apoyen la integración. de estas tecnologías en los planes de estudio y las prácticas pedagógica. Es de destacar que el largo

\footnotetext{
${ }^{1}$ Universidade do Vale do Sapucaí (UNIVÁS), Pouso Alegre - MG - Brasil. Professor do Programa de PósGraduação em Educação, Conhecimento e Sociedade. Doutorado em Ciências do Movimento Humano (UNIMEP). ORCID: https://orcid.org/0000-0003-4828-5212. E-mail: ronaldobaganha@univas.edu.br

${ }^{2}$ Universidade do Vale do Sapucaí (UNIVÁS), Pouso Alegre - MG - Brasil. Doutoranda no Programa de PósGraduação em Educação, Conhecimento e Sociedade. ORCID: https://orcid.org/0000-0003-3393-3256. E-mail: carolbrasil_pa@hotmail.com

${ }^{3}$ Universidade do Vale do Sapucaí (UNIVÁS), Pouso Alegre - MG - Brasil. Mestrando no Programa de PósGraduação em Educação, Conhecimento e Sociedade. ORCID: https://orcid.org/0000-0002-9546-7724. E-mail: lucasgambogi@yahoo.com.br
} 
tiempo de exposición a la "pantalla" puede perjudicar la salud, por lo que es necesario orientar a profesores y alumnos, en el sentido de prevenir daños a la salud mental.

PALABRAS CLAVE: Educación. Pandemia. TDIC. Tiempo de pantalla.

ABSTRACT: This article aimed to review, through the literature, the challenges and transformations imposed by the COVID-19 pandemic education, the main contributions of teacher education to innovation in the teaching and learning processes with the use of DICT and the possible health consequences of increased time of screen and high sedentary behavior. The results show that, given the challenges of education due to the pandemic, the domain and understanding of knowledge about the DICT used in the school environment are essential for teachers, which implies the need for training courses of teachers who support the integration of these technologies in curriculum and pedagogical practices. It is noteworthy that the long exposure "screen" time can harm health, and thus, it is necessary to provide guidance to teachers and students, in the sense of preventing damage to mental health.

KEYWORDS: Education. Pandemic. DICT. Time of screen.

\section{Introdução}

Em dezembro de 2019 na província Hubei em Wuhan, China foi identificada uma gripe responsável por causar uma síndrome denominada Síndrome Aguda Respiratória Severa (SARS), como consequência da doença causada pelo vírus corona (COVID), denominado assim como COVID-19 (ISER et al., 2020). Em janeiro de 2020, a Organização Mundial de Saúde declarou estado de Emergência em Saúde Pública de importância internacional, instalava-se neste momento a pandemia da COVID-19 (WHO, 2020).

Todas as pandemias são geradoras de forte impacto social, econômico e políticos e nesta não foi diferente. Com a pandemia declarada e a adoção de medidas de prevenção para conter seu avanço, os alunos foram impedidos de frequentar as aulas de forma presencial e em atendimento à portaria do Ministério da Educação (MEC), 343 de 17 de março de 2020, a educação foi direcionada do ensino tradicional presencial para o remoto (BRASIL, 2020).

A manutenção das atividades escolares só foi e continua sendo possível pelo uso das Tecnologias Digitais da Informação e Comunicação (TDIC), assim, o uso das TDIC foi ampliada e ganhou status em todos os segmentos da sociedade, incluindo o educacional (BRASIL, 2020). Nas instituições de ensino, os professores e os alunos tiveram que se adaptar aos novos recursos, como o uso das plataformas virtuais de ensino e aprendizagem, até então utilizados prioritariamente pelo Ensino a Distância (EaD), entretanto essa situação gerou grandes desafios e tensões para o segmento educacional (SANTANA; SALES, 2020, p. 
77), pois, a grande maioria das escolas, professores e alunos não estavam preparados para utilizar as TDIC e transformar o ambiente familiar em espaço de trabalho e estudo.

$\mathrm{O}$ uso dos ambientes virtuais de aprendizagem foi uma das alternativas que possibilitou a continuidade da educação em todo o mundo, porém, como consequência deste, observou-se um aumento no "tempo de tela" (time screen) (BOERS et al., 2019) e comportamento sedentário, com possíveis repercussões negativas à saúde, o que enaltece a necessidade de discussões nesta área.

Essas considerações iniciais enaltecem a necessidade de discussão sobre qual caminho a Educação e a formação de professores deve trilhar para favorecer a inserção dos futuros profissionais da Educação neste processo de inovação das práticas pedagógicas e a inserção das TDIC no ambiente escolar, e ainda qual o possível impacto do uso das novas tecnologias, do tempo de tela e do elevado comportamento sedentário sobre à saúde.

Assim, o objetivo do presente estudo foi revisar na literatura os desafios e as transformações impostas pela pandemia da COVID-19 sobre à educação, as principais contribuições da formação de professores para inovação no processo de ensino-aprendizagem com a utilização das TDIC e as possíveis consequências à saúde do aumento do tempo de tela e elevado comportamento sedentário.

\section{Percurso metodológico}

Para responder o objetivo proposto, o presente estudo apropriou-se metodologicamente na pesquisa de revisão bibliográfica, que segundo Severino (2016, p. 102), define por:

[...] aquela que realiza a partir do registro disponível, decorrente de pesquisas anteriores, em documentos impressos, como livros, artigos, teses etc.

As bases de dados consultadas foram Scielo, PubMed, Google Acadêmico e Bireme. As palavras chaves utilizadas em língua portuguesa, foram: formação docente; Educação; Educação a Distância; COVID-19 e TDIC, e na língua inglesa Education, Distance Education, COVID-19 e DICT. Para a pesquisa as palavras chaves foram utilizadas de forma isolada e/ou em expressões com o uso das conjunções "e/ou”. O período de busca, leitura e seleção dos materiais foi de maio de 2020 a junho de 2021. Foram selecionados artigos que tratavam da formação de professores, do uso das TDIC na escola e os que apresentavam os efeitos do aumento do tempo de exposição a tela e elevado comportamento sedentário à saúde. Foram 
excluídos artigos que após a leitura do resumo não contemplavam a relação com o processo formativo de futuros profissionais da Educação, a inserção das TDIC e discussão sobre as consequências do tempo de tela e elevado comportamento sedentário à saúde.

\section{TDIC na educação}

Nos últimos anos tem-se presenciado significativas alterações nos diferentes segmentos da sociedade, sendo estas impulsionadas pela ampliação do acesso às TDIC. A popularização das tecnologias e a evolução do uso das TDIC favoreceram a incorporação das redes digitais nos sistemas de comunicação e ações cotidianas (KENSKI, 2015).

Valente (2014, p. 79) afirma que John Dewey em seu livro "A democracia da Educação", publicado nos Estados Unidos da América há mais de um século, criticou o ensino-aprendizagem baseados apenas na transmissão de conhecimento e informação. $\mathrm{O}$ autor propôs uma educação baseada no interesse e experiências que os alunos traziam para a sala de aula, tornando-os protagonistas de sua aprendizagem. Segundo Borges e Oliveira (2020) em 1932 os educadores no Brasil influenciados pela teoria de Dewey, formaram um movimento conhecido como Escola Nova, ao qual pretendia que a formação do aluno fosse humanizada e que esses participassem de forma ativa nas atividades, consolidando sua aprendizagem.

Além de Dewey, outros estudiosos e educadores, como os norte-americanos Carl Ransom Rogers e Joseph Donald Novack, e o brasileiro Paulo Freire, reforçavam o quão relevante seria substituir a educação tradicional por uma onde o aluno fosse o protagonista de seu aprendizado, com o professor apenas envolvendo-o e motivando-o, sendo para isso necessário:

[...] a criação de desafios, atividades, jogos que realmente trazem as competências necessárias para cada etapa, que solicitam informações pertinentes, que oferecem recompensas estimulantes, que combinam percursos pessoais com participação significativa em grupos, que se inserem em plataformas adaptativas, que reconhecem cada aluno e ao mesmo tempo aprendem com a interação, tudo isso utilizando as tecnologias adequadas (MORAN, 2015, p. 18).

Quando se trata de tecnologias na educação, Valente (2013) cita ser necessário fazer integração das TDIC no currículo escolar, pois, o professor terá o papel de agente da aprendizagem, atuando como mediador entre a tecnologia e o conteúdo, assim, este necessita conhecer a funcionalidade educativa dos computadores, celulares e da internet, como também, 
ter domínio das ferramentas que podem ser utilizadas durante as aulas, interagindo com os alunos e atuando como mediador da aprendizagem.

Segundo Costa (2013), para que exista a integração entre as tecnologias e os conteúdos escolares é necessário a presença das TDIC na formação dos professores, pois, estas capacitam os mesmos para atuar com as tecnologias e enfrentar os desafios escolares, como os impostos pela pandemia da COVID-19. De acordo com Alonso et. al (20140 para ensinar com tecnologias é preciso aprender com as tecnologias, discutir coletivamente estratégias de reconstrução da ação do professor em um movimento permanente, onde todos se beneficiam.

O acesso às tecnologias e a utilização delas pode alterar a dinâmica da aula, e provocar mudanças pedagógicas. Desta forma, Valente (2013, p. 42) declara ser evidente os "aspectos inovadores relacionados ao uso das TDIC na educação". Para que a inovação na escola ocorra é essencial que as práticas pedagógicas, assim como a ação docente, alcancem novas dimensões no que diz respeito a criar um ambiente propício para comunicação, interação, resolução de problemas, de maneira interdisciplinar, com o intuito de estimular o raciocínio e o desenvolvimento dos alunos (ANDRADE, 2013). Nesse cenário, com a ajuda do professor, os alunos poderão desenvolver suas potencialidades por meio da interação e compartilhamento na construção do conhecimento, construindo novas competências para conseguirem alcançar a autonomia individual e coletiva.

O ensino e a aprendizagem ocorrem "numa interligação simbiótica constante" entre o "mundo físico e mundo digital", onde não se trata de "dois mundos ou espaços, mas sim de um espaço estendido, uma sala de aula ampliada, que se mescla e se hibridiza constantemente". Dessa forma, a educação está se tornando "cada vez mais híbrida, porque não acontece só no espaço físico da sala de aula, mas nos múltiplos espaços do cotidiano, que incluem os digitais" (MORAN, 2015, p. 16). Os professores, além do contato presencial, continuarão comunicando-se com seus alunos por meio de espaços digitais, estendendo a sala de aula para outros ambientes e espaços, tornando o processo de ensino-aprendizagem mais amplo, profundo e perceptível, com mobilidade e conectividade (BACICH; MORAN, 2015).

\section{Formação docente}

Ao analisar o papel do professor em relação ao processo de ensino, percebe-se que sua atuação vai além do ensinar (BERALDO; MACIEL, 2016), envolve uma função social, dialética, construtiva e transformadora. Segundo Moore (2019), faz-se necessário olhar o "professor" como um profissional que necessita de constante atualização e vivência, haja vista 
que o ato de ensinar é capaz de transferir muito mais do que conhecimento, mas também experiências.

No Brasil, a formação de profissionais da "Educação" segue normativas e orientações do Estado. A Lei no 9.394, de 20 de dezembro de 1996, conhecida como Lei de Diretrizes e Bases da Educação Nacional (LDBN), estabelece em seu Art. 62 que os cursos de formação de professores para atuarem na educação podem ser presenciais ou a distância (BRASIL, 1996). Nos parágrafos 2 e 3, deste mesmo artigo é apresentado sobre o uso dos recursos tecnológicos:

A formação continuada e a capacitação dos profissionais de magistério poderão utilizar recursos e tecnologias de educação a distância. (Incluído pela Lei $\mathrm{n}^{\mathrm{o}}$ 12.056, de 2009). A formação inicial de profissionais de magistério dará preferência ao ensino presencial, subsidiariamente fazendo uso de recursos e tecnologias de educação a distância. (Incluído pela Lei ${ }^{\circ}$ 12.056, de 2009) (BRASIL, 1996).

A Resolução CNE/CP, $\mathrm{n}^{\mathrm{o}}$ 2, de 20 de dezembro de 2019, define as Diretrizes Curriculares Nacionais para a Formação Inicial de Professores para a Educação Básica e institui a Base Nacional Comum para a Formação Inicial de Professores da Educação Básica (BNC-Formação), e em seu anexo são apresentadas as competências gerais docentes e a necessidade pela busca de "soluções tecnológicas para uma evolução das práticas pedagógicas" (BRASIL, 2019, p. 13). As TDIC estão também presentes na ação de planejamento e ensino, as quais devem subsidiar a aprendizagem, e ao docente cabe incorporar seu uso em sua prática pedagógica, com o objetivo de favorecer a aprendizagem e transformar a educação (BRASIL, 2020).

Dentre os modelos de formação de professores, descritos por Jacobucci (2006), incluem-se o modelo clássico, que consiste em atualizações concebidas pelo modelo ensinoaprendizagem; o modelo prático reflexivo, que contempla as práticas e experiências dos professores e o modelo emancipatório-político, no qual a pesquisa-ação é utilizada como uma estratégia de aprendizagem. E além destes modelos, destaca-se outro movimento utilizado para a formação do professor, com o uso das TDIC, que tem se mostrado necessário, porém desafiador (SIQUEIRA; MOLON; FRANCO, 2021).

Strycer (2021) apresenta que o professor para estar apto a usar as TDIC como uma ferramenta, precisa de cursos de integração com estas tecnologias. Em relação aos cursos de formação de professores em instituições de Ensino Superior (IES) segundo Valente, Almeida e Geraldini (2017), necessitam proporcionar situações de aprendizagem que integrem as 
TDIC as suas atividades práticas para que esses futuros professores possam experienciar situações de ensino e de aprendizagem fundamentados nessas tecnologias.

$\mathrm{Na}$ contemporaneidade, os professores precisam ter competências e habilidades para conduzir suas práticas pedagógicas por meio das TDIC (BRASIL, 2019), pois o uso das TDIC na educação, principalmente pelo uso de celulares, smartphones e notebooks possibilitam uma transformação dos conceitos de espaço e tempo (BERALDO; MACIEL, 2016).

Misha e Koehler (2006) ao perceberem a necessidade de uma teoria que auxiliasse na formação de professores para o uso das TDIC, criaram a Technological Pedagogical Content Knowledge (TPACK), traduzida em português como "Conhecimento do conteúdo, pedagógico e tecnológico". Essa teoria tem por objetivo auxiliar os professores na compreensão de suas aulas, ao reconhecer os contextos e interações que estão envolvidos nos processos de ensino e de aprendizagem, sendo a representação da integração entre o conhecimento de conteúdo, pedagógico e tecnológico.

A introdução das TDIC nas aulas é um processo complexo (ALMEIDA, 2018) e dessa forma estas não devem ser utilizadas de forma isolada, mas sim associadas ao conhecimento pedagógico do professor e aos conteúdos curriculares, favorecendo a dinamicidade e a interação entre professores e alunos. Nessa direção, a descrição de práticas incorporadas às TDIC tem sido crescente e a inserção do contexto tecnológico tem contribuído para o desenvolvimento da competência digital (CERVERA; MARTÍNEZ; MON, 2016), onde se observa um aumento no número de professores utilizando computadores, softwares e da internet em sua prática pedagógica (COSTA, 2020). Assim, a integração das TDIC nas práticas dos professores tem sido tema de grande interesse nas pesquisas, pois, as tendências tecnológicas apontam para uma influência destas na forma de educar (SIQUEIRA; MOLON; FRANCO, 2021).

Pode-se assim observar que a integração das TDIC alcançou as mais diversas áreas da educação (GUZE, 2015). Mas como preparar os futuros e atuais professores para integrar as TDIC as aulas? Alelamait, Ihmeideh e Alkhawaldeh (2021) descrevem que a oferta de cursos para professores na área de tecnologias tende a ser eficiente, pois, favorecem o aprendizado e permitem colocar o conhecimento e as habilidades em prática.

Avaliando o cenário educativo por essa vertente, acredita-se que o conhecimento sobre recursos tecnológicos passou a ser uma obrigação e emergente nos cursos de formação docente, pois cada dia mais o aluno chega "mais" digital. 


\section{Educação em tempos de pandemia}

Entre os impactos da pandemia da COVID-19 no âmbito educacional no Brasil, estão a suspensão das atividades presenciais e a utilização de alternativas para manutenção do processo de ensino-aprendizagem, em sua maioria com o uso das TDIC, que tem o objetivo de preservar o direito de todos à educação e reduzir o prejuízo educacional associado a pandemia (FCC, 2020).

Dentro desse contexto, conforme uma pesquisa realizada pela Fundação Carlos Chagas (FCC) com a participação do Itaú social e da Unesco, em 2020, desde que as medidas de isolamento foram implantadas no Brasil, cerca de 39 milhões de alunos $(81,9 \%)$ que frequentavam a educação básica foram afastados das cadeiras escolares (FCC, 2020). Além disso, dados da Unesco (2020) apontam que inúmeras instituições de ensino foram prejudicadas pelo contexto da pandemia, resultante do fechamento, deixando sem aulas, milhares de alunos.

Segundo, Aguiar, Paniago e Cunha (2020), os professores tiveram em pouco tempo, uma grande mudança em seu cotidiano escolar, com dificuldades e poucos recursos, foram impulsionados a se adaptar ao uso das TDIC para continuidade de suas atividades profissionais e interações com os alunos, e o que antes era utilizado apenas para complementação de material pedagógico, tornou-se uma ferramenta necessária em tempos de pandemia da COVID-19, e com os alunos as dificuldades não foram diferentes.

Entretanto, sabe-se que a dificuldade ao acesso destas tecnologias, impactou a educação de forma abrupta, trazendo prejuízo ao ensino, devido a desigualdade social. $\mathrm{O}$ impacto pandêmico também assolou aos estudantes, que muitos, por sua vez, não tinham acesso à internet e as tecnologias como computadores ou celulares, recursos esses, imprescindíveis para o acesso as aulas, dificultando o seu aprendizado. Alguns dependiam, prioritariamente, das redes educacionais, para que pudessem acompanhar as aulas (GATTI, 2020).

Frente a essa situação pandêmica, as escolas públicas e privadas, criam expectativas quanto ao trabalho do professor, para que este mantenha a qualidade de suas atividades pedagógicas durante o período de isolamento, entretanto, muitas famílias não conseguem atender a este contexto, haja vista que muitos pais não conseguem acompanhar e estimular o ensino de seus filhos, seja pela falta de recursos tecnológicos ou também pelo desnivelamento na educação dos próprios pais (DIAS; PINTO, 2020). 
Uma prática que se tornou comum neste momento vivido, foi a adoção de aulas em sistema híbrido, sistema esse, que compõe uma mistura de aulas remotas com aulas presenciais, mantendo a interação por meio das tecnologias (MEDEIROS, et al, 2020). Uma pesquisa realizada no estado de São Paulo, pela Universidade de São Paulo, com 19.221 professores da rede estadual, aponta que 70\% responderam sentir-se aptos no desempenho do seu trabalho utilizando essas tecnologias, e destes, $51 \%$ responderam sentir-se inseguros para utilizá-las em suas aulas (GRANDISOLI; JACOBI; MARCHINI, 2020).

Segundo Silva (2020), existe um desejo dos professores em aprender a utilizar os recursos digitais, com uma determinada urgência, para que haja a adaptação ao momento, como um aspecto essencial e importante para a formação continuada. Entretanto, só esse desejo não basta, é necessário reconhecer que ainda se tem obstáculos a serem vencidos, por ambos os lados, de um, os centros educacionais, que sofrem com a falta de recursos tecnológicos e deixam de oferecer componentes curriculares, como as aulas de laboratórios, estágios, entre outros, e os alunos, que também enfrentam a falta de acesso aos recursos tecnológicos e vivenciam a dificuldade em aprender sozinhos, principalmente para aqueles que os pais ou responsáveis não podem acompanhá-los nas aulas ou no fazer das atividades remotas (GATTI, 2020; DIAS; PINTO, 2020)

\section{Saúde em tempos de pandemia e a influência do tempo de tela e do elevado comportamento sedentário}

Com a declaração da pandemia pela Organização Mundial da Saúde (WHO, 2020), governantes do mundo todo publicaram decretos para o fechamento do comércio, escolas, academias e orientaram a população a permanecer em casa. As atividades do cotidiano como ir as compras, à escola, participar de reuniões, passaram de uma condição prática para on line, com impactos sem precedentes ao comportamento humano (VARGO et al., 2021).

O aumento das atividades on line como consequência da pandemia da COVID-19, favoreceu a elevação do tempo de tela, e aqui destaca-se as atividades escolares. Com o aumento no tempo de tela, foi observado aumento nos episódios de stress, ansiedade (LEE SMITH et al., 2020), depressão, diminuição dos níveis de atividades físicas e aumento do comportamento sedentário (GIUNTELLA et al., 2021), sendo cada um destes responsáveis por prejuízos à saúde.

Segundo a WHO (2017) os transtornos mentais são comuns em todas as idades, no entanto, durante a adolescência associa-se ao comprometimento acadêmico (VERBOOM et 
al., 2014) e cognitivo (ROCK, 2014), o que pode comprometer a saúde mental não só de crianças e adolescentes, mas também de adultos.

No estudo Wang et al. (2020), realizado com 1.210 participantes de 21 a 30 anos, em 194 cidades na China, 53,8\% relataram impacto psicológico da COVID-19 como moderado ou severo, relatando sintomas moderados ou severos de ansiedade (28,8\%), depressão $(16,5 \%)$ e estresse $(8,1 \%)$. Em outro trabalho foi demonstrado aumento significativo de perturbação psicológica (ansiedade, depressão e estresse) entre os estudantes universitários no período pandêmico comparativamente a períodos não pandêmicos (MAIA; DIAS, 2020).

O tempo de tela e o elevado comportamento sedentário associam-se a uma série prejuízos à saúde mental e física (ALLEN; WATTER; SWANN, 2019; HUANG et al., 2020). A prática regular de atividades físicas é fundamental para saúde (SEVERINSEN; PERDERSEN, 2020) e prevenção de doenças (FEALY et al., 2018), sendo a inatividade física relacionada ao desenvolvimento de várias doenças crônicas não transmissíveis (MCGEE; HARGREAVES, 2020), e considerada como um dos maiores problemas modificáveis (BOOTH; ROBERTS; LAYE, 2012) de saúde pública do século XXI (BLAIR, 2009).

O Colégio Americano de Medicina Esportiva (ACSM, 2020) sugeriu que durante a pandemia da COVID-19, as pessoas continuassem com a prática regular de atividades físicas, reconhecendo a mesma como essencial à saúde. Pesquisadores brasileiros fizeram a mesma colocação, e chamaram atenção sobre a importância da prática regular da atividade em casa ou ao ar livre, enfatizando, também, a importância da redução dos episódios de comportamentos sedentários (PITANGA; BECK; PITANGA, 2020).

Para a prevenção das perturbações psicológicas e seus sintomas têm sido recomendado redução do tempo de tela (BOERS et al., 2019) e aumento nos níveis de atividades físicas, mas nos parece que a ausência de informações sobre estas questões ainda prevalece, o que torna difícil, a curto prazo, a reversão destes problemas.

\section{Considerações finais}

O objetivo do presente estudo foi revisar por meio da literatura os desafios e as transformações impostas pela pandemia da COVID-19 à educação, as principais contribuições da formação docente para inovação nos processos de ensino e aprendizagem com a utilização das TDIC e as possíveis consequências à saúde do aumento do tempo de tela e elevado comportamento sedentário. 
Considerando os desafios impostos a educação pela pandemia da COVID-19, como a utilização das TDIC, a necessidade de manutenção da qualidade do processo de ensino e aprendizagem no formato remoto, entre outros, considera-se ser necessário por parte dos professores o domínio das TDIC, e para tanto, é necessário que os cursos de formação adicionem em suas práticas e conteúdos pedagógicos o uso das TDIC. Destaca-se ainda que o elevado tempo de exposição à tela e os elevados episódios de comportamento sedentário, como os observados neste momento de pandemia podem prejudicar a saúde física e mental, sendo necessário e importante que o Ministério da Saúde, da Educação e de maneira geral de todos os profissionais da saúde se posicionem sobre estas situações.

\section{REFERÊNCIAS}

ACSM. American College of Sport Medicine. Staying Physically Active During the COVID-19 Pandemic. Disponível em: https://www.acsm.org/read-research/newsroom/newsreleases/news-detail/2020/03/16/staying-physically-active-during-covid-19-pandemic. Acesso em: 20 maio 2021.

AGUIAR, L.; PANIAGO, R. N.; CUNHA, F. S. R. Os impactos do coronavírus no saber fazer docente dos professores do ensino médio integral. Itinerarius Reflectionis, v. 16, n. 1, p. 01-22, 2020. Disponível em: https://www.revistas.ufg.br/rir/article/view/65352. Acesso em: 20 maio 2021.

ALELAMAIT, A. M.; IHMEIDEH, F. M.; ALKHAWALDEH, M. F. Preparing preservice teachers for technology and digital media integration: implications for early childhood teacher education programs. International Journal of Early Childhood, v. 52, n.3, p. 299-317, jan. 2021. Disponível em:

https://www.ncbi.nlm.nih.gov/pmc/articles/PMC7811685/pdf/13158_2020_Article_276.pdf. Acesso em: 20 maio 2021.

ALLEN, M. S.; WALTER, E. E.; SWANN, C. Sedentary behaviour and risk of anxiety: a systematic review and meta-analysis. Journal of Affective Disorders, v. 242, p. 5-13, 2019. Disponível em: https://www.sciencedirect.com/science/article/abs/pii/S0165032718305299. Acesso em: 20 maio 2021.

ALMEIDA, P. Tecnologias digitais em sala de aula: o professor e a reconfiguração do processo educativo. Da Investigação às Práticas, v. 8, n.1, p. 4-21, 2018. Disponível em: http://www.scielo.mec.pt/pdf/inp/v8n1/v8n1a02.pdf. Acesso em: 20 maio 2021.

ALONSO, K. M. et al. Aprender e ensinar em tempos de cultura digital. Em Rede Revista de Educação a Distância, Porto Alegre, v. 1, n. 1, p. 152-168, 2014. Disponível em: https://www.aunirede.org.br/revista/index.php/emrede/article/view/16. Acesso em: 20 maio 2021.

ANDRADE, P. F. Inovação em TIC na educação no Brasil: sustentabilidade e mudança pedagógica. In: ALMEIDA, M. E. B.; DIAS, P.; SILVA, B. D. (org.). Cenários educativos 
de inovação digital. São Paulo: Edições Loyola, 2013.

BACICH, L.; MORAN, J. Aprender e ensinar com foco na educação. Revista Pátio, n. 25, p. 45-47, jun. 2015.

BASSOLI, F.; LOPES, J. G. S.; SILVA, E. T. C. Reflexões sobre experiências de formação continuada de professores em um centro de ciências: trajetória, concepções e práticas formativas. Ciência \& Educação, Bauru, v. 23, n. 4, p. 817-834, 2017. Disponível em: https://www.scielo.br/j/ciedu/a/5pfQLg6FWgQ7SXc5rhNJv4y/?lang=pt. Acesso em: 20 maio 2021.

BERALDO, R. M. F.; MACIEL, D. A. Competência do professor no uso das TDIC e de ambientes virtuais. Psicologia Escolar e Educacional, São Paulo, v. 20, n. 2, p. 209-217, maio/ago. 2016.

BLAIR, S. N. Physical inactivity: the biggest public health problem of the 21 st century. British journal of sports medicine, v. 43, n. 1, p. 1-2, 2009. Disponível em: https://bjsm.bmj.com/content/43/1/1.short. Acesso em: 20 maio 2021.

BOERS, E. et al. Association of screen time and depression in adolescence. JAMA Pediatrics, v. 173, n. 9, p. 853-859, 2019. Disponível em: https://jamanetwork.com/journals/jamapediatrics/fullarticle/2737909. Acesso em: 20 maio 2021.

BOOTH, F. W.; ROBERTS, C. B. K.; LAYE, M. J. Lack of exercise is a major cause of chronic diseases. Comprehensive Physiology, v. 2, n. 2, p. 1143-1211, 2012. Disponível em: https://www.ncbi.nlm.nih.gov/pmc/articles/PMC4241367/. Acesso em: 20 maio 2021.

BORGES, R. A. S.; OLIVEIRA, A. L. M. O. Tecnologias digitais no ensino superior: inovação com a TPACK. Curitiba: Appris, 2020. 143 p.

BRASIL. Lei n. 9.396, de 20 de dezembro de 1996. Estabelece as diretrizes e bases da educação nacional. Brasília, DF, 23 dez. 1996. Disponível em:

http://www.planalto.gov.br/ccivil_03/leis/19394.htm. Acesso em: 20 maio 2021.

BRASIL. Ministério da Educação. Portaria n. 343 de 17 de março de 2020. Dispõe sobre a substituição das aulas presenciais por aulas em meios digitais enquanto durar a situação de pandemia do Novo Coronavírus - COVID-19. Brasília, DF: MEC, 18 mar. 2020. Disponível em https://abmes.org.br/legislacoes/detalhe/3017/portaria-mec-n-343. Acesso em: 20 maio 2021.

BRASIL. Resolução CNE/CP n. 2, de 20 de dezembro de 2019. Define as Diretrizes Curriculares Nacionais para a Formação Inicial de Professores para a Educação Básica e institui a Base Nacional Comum para a Formação Inicial de Professores da Educação Básica (BNC-Formação). Brasília, DF, 10 fev. 2020. Disponível em:

http://portal.mec.gov.br/docman/dezembro-2019-pdf/135951-rcp002-19/file. Acesso em: 20 maio 2021.

CERVERA, M. G.; MARTÍNEZ, J. G.; MON, F. M. E. Competencia digital y competencia digital docente: una panorámica sobre el estado de la cuestión. RIITE: Revista 
Interuniversitaria de Investigación en Tecnología Educativa, n. 0, p. 74-83, 2016.

Disponível em: https://revistas.um.es/riite/article/view/257631. Acesso em: 20 maio 2021.

COSTA, F. A. O potencial transformador das TIC e a formação de professores e educadores. In: ALMEIDA, M. E. B. de; DIAS, P.; SILVA, B. D. (org.). Cenários de inovação para a educação na sociedade digital. São Paulo: Edições Loyola, 2013.

COSTA, R. T. O. Formação docente: inovar é preciso. In: CONGRESSO INTERNACIONAL DE EDUCAÇÃO E TECNOLOGIAS | ENCONTRO DE PESQUISADORES EM EDUCAÇÃO A DISTÂNCIA, 2020, São Carlos. Anais [...]. São Carlos, SP: UFSCAR, 2020. ISSN 2316-8722. Disponível em:

https://cietenped.ufscar.br/submissao/index.php/2020/article/view/1760. Acesso em: 20 maio 2021.

DIAS, E.; PINTO, F. C. A Educação e a COVID-19. Ensaio: Aval. Pol. Publi. Educ., Rio de Janeiro, v. 28, n. 108, p. 545-554, jul./set. 2020.

FCC. FUNDAÇÃO CARLOS CHAGAS. Pesquisa: educação escolar em tempos de pandemia na visão de professoras/es da Educação Básica: informe 1. 2020. Disponível em: https://www.fcc.org.br/fcc/wp-content/uploads/2020/06/educacao-pandemia-a4_1606_final.pdf. Acesso em: 20 maio 2021.

FEALY, C. E. et al. Functional high-intensity exercise training ameliorates insulin resistance and cardiometabolic risk factors in type 2 diabetes. Experimental Physiology, v. 103, n. 7, p. 985-994, 2018. Disponível em:

https://physoc.onlinelibrary.wiley.com/doi/full/10.1113/EP086844. Acesso em: 20 maio 2021.

GATTI, B.A. Possível Reconfiguração dos Modelos Educacionais pós pandemia. Estudos Avançados, v. 34, n. 100, set./dez. 2020 Disponível em:

https://www.scielo.br/j/ea/a/7M6bwtNMyv7BqzDfKHFqxfh/?lang=pt Acesso em: 20 maio 2021.

GIUNTELLA, O. et al. Lifestyle and mental health disruptions during COVID-19.

Proceedings of the National Academy of Sciences, v. 118, n. 9, 2021. Disponível em: https://www.pnas.org/content/118/9/e2016632118.short. Acesso em: 20 maio 2021.

GRANDISOLI, E.; JACOBI, P. R; MARCHINI, S. Pesquisa Educação, Docencia e a COVID-19. Programa USP Cidades Globais, São Paulo, 2020. Disponível em: http://www.iea.usp.br/pesquisa/projetos-institucionais/usp-cidades-globais/pesquisaeducacao-docencia-e-a-covid-19. Acesso em: 20 maio 2021.

GUZE, P. A. Using technology to meet the challenges of medical education. Transactions of the American Clinical and Climatological Association, v. 126, p. 260-270, 2015. Disponível em: https://www.ncbi.nlm.nih.gov/pmc/articles/PMC4530721/. Acesso em: 20 maio 2021.

HUANG, Y. et al. Sedentary behaviors and risk of depression: a meta-analysis of prospective studies. Translational psychiatry, v. 10, n. 1, p. 1-10, 2020. Disponível em: https://www.nature.com/articles/s41398-020-0715-z. Acesso em: 20 maio 2021. 
ISER, B. P. M. et al. Definição de caso suspeito da COVID-19: uma revisão narrativa dos sinais e sintomas mais frequentes entre os casos confirmados. Revista Epidemiologia e Serviços de Saúde, Brasília, v. 29, n. 3, p. 1-11, 2020. Disponível em:

https://www.scielosp.org/article/ress/2020.v29n3/e2020233/. Acesso em: 20 maio 2021.

\section{JACOBUCCI, D. F. C. A formação continuada de professores em centros e museus de}

ciências no Brasil. 2006. 302 f. Tese (Doutorado em educação) - Universidade Estadual de Campinas, Campinas, 2006. Disponível em:

http://www.museudavidahomolog.fiocruz.br/brasiliana/media/Tese_Jacobucci.pdf. Acesso em: 20 maio 2021.

KENSKI, V. M. A urgência de propostas inovadoras para a formação de professores para todos os níveis de ensino. Revista Diálogo Educacional, v. 15, n. 45, p. 423-441, 2015. Disponível em: https://periodicos.pucpr.br/index.php/dialogoeducacional/article/view/1963. Acesso em: 20 maio 2021.

LEE SMITH, L. J. et al. The association between screen time and mental health during COVID-19: a cross sectional study. Psychiatry research, v. 292, p. 1-2, 2020. Disponível em: https://www.ncbi.nlm.nih.gov/pmc/articles/PMC7382341/. Acesso em: 20 maio 2021.

LEITE, E. P. et al. Alguns desafios e demandas da formação inicial de professores na contemporaneidade. Educação e Sociedade, Campinas, v. 39, n. 144, p. 721-737, jul./set. 2018 .

MAIA, B. R.; DIAS, P. C. Ansiedade, depressão e estresse em estudantes universitários: o impacto da COVID-19. Estudos de Psicologia, Campinas, v. 37, e200067, 2020.

MCGEE, S. L.; HARGREAVES, M. Exercise adaptations: molecular mechanisms and potential targets for therapeutic benefit. Nature Reviews Endocrinology, v. 16, n. 9, p. 495505, 2020. Disponível em: https://www.nature.com/articles/s41574-020-0377-1. Acesso em: 20 maio 2021.

MEDEIROS, M. C. L. et al. Tecnologias Digiatsi em Aulas do Ensino Fundamental, Tecnia, Goiânia, v. 5, n. 2, p. 52-71, jul./dez. 2020.

MISHRA, P.; KOEHLER, M. J. Technological pedagogical content knowledge: a framework for teacher knowledge. Teachers College Record, v. 108, n. 6, p. 1017-1054, 2006.

MOORE, S. Language and identity in an Indigenous teacher education program.

International Journal of Circumpolar Health, v. 78, n. 2, p. 1-8, 2019. Disponível em https://www.tandfonline.com/doi/full/10.1080/22423982.2018.1506213. Acesso em: 20 maio 2021.

MORAN, J. Mudando a educação com metodologias ativas. In: SOUZA, C. A.; MORALES, O. E. T. (org.). Convergências midiáticas, educação e cidadania: aproximações jovens. Ponta Grossa: UEPG, 2015. Disponível em: http://www2.eca.usp.br/moran/wpcontent/uploads/2013/12/mudando_moran.pdf. Acesso em: 20 maio 2021. 
PITANGA F. J. G.; CARMEM, C. C.; PITANGA, C. P. S. Atividade física e redução do comportamento sedentário durante a pandemia do Coronavírus. Arquivos Brasileiros de Cardiologia, v. 114, n. 6, p. 1058-1060, 2020.

ROCK, P. L. et al. Cognitive impairment in depression: a systematic review and metaanalysis. Psychological medicine, v. 44, n. 10, p. 2029-2040, 2014.

SANTANA, C. L. S.; SALES, K. M. B. Aula em casa: educação, tecnologias digitais e pandemia Covid-19. Interfaces Científicas-Educação, v. 10, n. 1, p. 75-92, 2020. Disponível em: https://periodicos.set.edu.br/educacao/article/view/9181. Acesso em: 20 maio 2021.

SEVERINO, A. J. Metodologia do trabalho científico. São Paulo: Cortez, 2016.

SEVERINSEN, M. C. K.; PEDERSEN, B. K. Muscle-organ crosstalk: the emerging roles of myokines. Endocrine reviews, v. 41, n. 4, p. 594-609, 2020. Disponível em: https://academic.oup.com/edrv/article/41/4/594/5835999?login=true. Acesso em: 20 maio 2021.

SILVA, S. O que você e sua escola aprenderam nesta quarentena? Petrópolis: Vozes, 2020.

SIQUEIRA, C. F. R.; MOLON, J.; FRANCO, R. K. Professores de TDIC em um curso de licenciatura em matemática: desafios frente às tecnologias educacionais digitais. Ensino da Matemática em Debate, v. 8, p. 42-60, 2021. Disponível em:

https://revistas.pucsp.br/emd/article/download/49147/pdf. Acesso em: 20 maio 2021.

SOARES, A. B. et al. Metodologias ativas: potencializando a aprendizagem na educação profissional e tecnológica. In: SEMINÁRIO INTERNACIONAL DE EDUCAÇÃO NO MERCOSUL, 18., 2018, Cruz Alta. Anais [...]. Cruz Alta, RS: Casa da Cultura Justino Martins, 2018.

STRYCKER, J. K-12 art teacher technology use and preparation. Heliyon, v. 6 n. 7, p. 1-12, 2020 em: https://www.ncbi.nlm.nih.gov/pmc/articles/PMC7338783/. Acesso em: 20 maio 2021.

UNESCO. United Nations Educational, Scientific and Cultural Organization. Impactos da COVID-19 na Educação. 2020.

VALENTE, J. A. As tecnologias e as verdadeiras inovações na educação. In: ALMEIDA, M. E. B.; DIAS, P.; SILVA, B. D. (org.). Cenários de inovação para a educação na sociedade digital. São Paulo: Edições Loyola, 2013.

VALENTE, J. A. Blended learning e as mudanças no ensino superior: a proposta da sala de aula invertida. Educar em Revista, Curitiba, v. 30, n. 4, p. 79-97, 2014. Disponível em: http://www.scielo.br/pdf/er/nspe4/0101-4358-er-esp-04-00079.pdf. Acesso em: 20 maio 2021.

VALENTE, J. A.; ALMEIDA, M. E. B.; GERALDINI, A. F. S. Metodologias ativas: das concepções às práticas em distintos níveis de ensino. Revista Diálogo Educacional, v. 17, n. 52, p. 455-478, 2017. Disponível em: https://www.redalyc.org/pdf/1891/189154955008.pdf. Acesso em: 20 maio 2021. 
VARGO, D. et al. Digital technology use during COVID-19 pandemic: a rapid review. Human Behavior and Emerging Technologies, v. 3, n. 1, p. 13-24, 2021. Disponível em: https://onlinelibrary.wiley.com/doi/full/10.1002/hbe2.242. Acesso em: 20 maio 2021.

VERBOOM, C. E. et al. Longitudinal associations between depressive problems, academic performance, and social functioning in adolescent boys and girls. Developmental Psychology, v. 50, n. 1, p. 247-257, 2014. Disponível em: https://psycnet.apa.org/doiLanding?doi=10.1037\%2Fa0032547. Acesso em: 20 maio 2021.

WANG, C. et al. Immediate psychological responses and associated factors during the initial stage of the 2019 coronavirus disease (COVID-19) epidemic among the general population in China. International Journal of Environmental Research and Public Health, v. 17, n. 5 , p. 1-25, 2020. Disponível em: https://www.mdpi.com/1660-4601/17/5/1729. Acesso em: 20 maio 2021.

WHO. World Health Organization. Depression and Other Common Mental Health Disorders. Geneva, Switzerland, 2017. p. 1-17. Disponível em: https://apps.who.int./iris/bitstrean/hndle/10665/254610/WHO-MSD-MER-2017.2eng.pdf;sequence=1. Acesso em: 20 maio 2021.

WHO. World Health Organization. Guidelines on physical activity and sedentary behaviour. Geneva, Switzerland, 2020. Disponível em: http://www.WHO guidelines on physical activity and sedentary behaviour. Acesso em: 20 maio 2021.

WHO. World Health Organization. Novel Corona Vírus (2019-nCoV). Situation report: 1. Geneva, Switzerland, 21 jan. 2020.

WHO. World Health Organization. WHO Director-General's remarks at the media briefing on 2019-nCoV on 11 February 2020. Geneva, Switzerland, 11 fev. 2020. Disponível em: https://www.who.int/dg/speeches/ detail/whodirector-general-s-remarks-atthe-media-briefing-on-2019-ncov-on-11-february-2020. Acesso em: 20 maio 2021.

\section{Como referenciar este artigo}

BAGANHA, R. J.; BERNARDES, A. C. B.; ANTUNES, L. G. Educação, formação docente, TDIC e saúde em tempos de pandemia pela COVID-19: uma revisão de literatura. Temas em Educ. e Saúde, Araraquara, v. 17, n. 00, e021017, 2021. e-ISSN 2526-3471. DOI: https://doi.org/10.26673/tes.v17i00.15261

Submetido em: 13/07/2021

Revisões requeridas: 25/07/2021

Aceito em: 11/08/2021

Publicado em: 19/08/2021 